\title{
NUEVAS LOCALIDADES Y FORMAS FLORALES DEL ENDEMISMO MEXICANO STELIS ZOOTROPHIONOIDES (ORCHIDACEAE), Y EVALUACIÓN DE SU RIESGO DE EXTINCIÓN
}

\author{
Alfredo García-González ${ }^{1,5}$, Frander B. Riverón-Giró ${ }^{1}$, Rodolfo Solano-Gómez², \\ Osiris Aguilar-Romero ${ }^{3}$ y Eduardo Martínez-OVando ${ }^{4}$ \\ 'El Colegio de la Frontera Sur, Unidad Tapachula, Tapachula, Chiapas, México. \\ ${ }^{2}$ Instituto Politécnico Nacional, Centro Interdisciplinario de Investigación para el Desarrollo Integral Regional, \\ Unidad Oaxaca, Oaxaca, México. \\ ${ }^{3}$ Universidad Autónoma Metropolitana, Unidad Iztapalapa, Iztapalapa, México D.F. \\ ${ }^{4}$ Programa de Conservación Voluntaria de Tierras, Pronatura Sur A.C., San Cristóbal de Las Casas, Chiapas, México. \\ ${ }^{5}$ Autor para correspondencia: alfredmx22@gmail.com
}

\begin{abstract}
Resumen: Stelis zootrophionoides (Orchidaceae) fue descrita recientemente a partir de material recolectado en las inmediaciones de la ciudad de San Cristóbal de Las Casas, Chiapas, México. En este trabajo se informa del descubrimiento de nuevas localidades de S. zootrophionoides y se registran formas florales (basadas en la coloración de sus flores) no conocidas previamente para la ciencia. Se modela la distribución potencial de la especie y se evalúa su riesgo de extinción a través del Método de Evaluación del Riesgo de Extinción para plantas en México (MER-Plantas). Adicionalmente se caracteriza una de sus poblaciones por ser la que enfrenta mayor riesgo. Stelis zootrophionoides ahora se conoce de cinco localidades, con un área de distribución potencial de $292 \mathrm{~km}^{2}$. Se encontraron dos nuevas formas de la especie, una con flores púrpuras con manchas blancas y otra con flores amarilloverdoso ("albinas"). De acuerdo a la evaluación del MER-Plantas, S. zootrophionoides se encuentra en peligro de extinción (P). En la población evaluada se encontraron 41 individuos, y está seriamente amenazada principalmente por la degradación del hábitat y la expansión de la zona urbana. Se hace necesario realizar estudios demográficos y genéticos para esta especie de orquídea, así como emprender y consolidar acciones que permitan la conservación de sus poblaciones conocidas.
\end{abstract}

Palabras clave: Chiapas, distribución potencial, MER-Plantas, orquídeas, San Cristóbal de Las Casas, vulnerabilidad biológica

\begin{abstract}
Stelis zootrophionoides (Orchidaceae) was described from a plant collected in the vicinity of San Cristóbal de Las Casas, Chiapas, México. This paper reports the discovery of new localities of $S$. zootrophionoides and floral forms (based on the color of its flowers) not previously known to science. The potential distribution of the species was modeled and the extinction risk was assessed through Risk Assessment Method of Extinction for plants in México (MER-Plantas). In addition, the population at greatest risk of the species was characterized. Four populations of S. zootrophionoides were discovered. Stelis zootrophionoides is now known from five localities, with a potential distribution area of $292 \mathrm{~km}^{2}$. Two new forms of the species were found, one with purple flowers with white spots and one with yellow-green flowers ("albino"). According to the evaluation of the MER-Plantas, S. zootrophionoides is in danger of extinction (P). The population evaluated is composed by 41 individuals, and is seriously threatened by the habitat degradation and the expansion of urban areas. Demographic and genetic studies of this orchid species are necessary, and to strengthen and implement actions that enable the conservation of its known populations.
\end{abstract}

Key words: Biological vulnerability, Chiapas, MER-Plantas, orchids, potential distribution, San Cristóbal de Las Casas.

$\mathbf{O}$ rchidaceae es la familia con la mayor riqueza de especies de epífitas vasculares en el mundo (Fay y Chase, 2009). Sin embargo, las orquídeas exhiben una condición contradictoria, ya que conforman la familia de plantas con flores más numerosas del planeta, y a la vez es una de las más amenazadas (Dillon, 1977; IUCN y OSG, 2012). México, situado en el límite norte del trópico americano, alberga una notable diversidad de orquídeas y han sido registradas en el país alrededor de 1,260 especies (60\% son epifitas) y 170 géneros (Hágsater et al., 2005; Soto-Arenas et al., 
2007). De esta gran riqueza de orquídeas, aproximadamente 188 especies se encuentran incluidas en alguna categoría de riesgo en la Norma Oficial Mexicana para la flora y la fauna amenazadas (SEMARNAT, 2010).

Uno de los géneros de orquídeas que se encuentran en México es Stelis Sw., que incluye aproximadamente 1,000 especies de pequeñas orquídeas epifitas, de la subtribu Pleurothallidinae, distribuidas en todo el neotrópico (Luer, 2014), y que en el país está representado por 66 especies (Solano-Gómez y Salazar, 2007; Solano-Gómez, 2010; Solano-Gómez, 2011; Ramos-Castro et al., 2012; Solano-Gómez, 2014). Este es un grupo monofilético fácilmente reconocible por su relativamente constante morfología floral (Solano-Gómez y Salazar, 2007).

Una de las especies de orquídeas mexicanas incluidas en este género es Stelis zootrophionoides Castañeda-Zárate \& Ramos-Castro (Figura 1A, B, C, D), descrita en 2012 a partir de una planta recolectada en el estado de Chiapas, en las inmediaciones de la ciudad de San Cristóbal de Las Casas (Ramos-Castro et al., 2012). En esta región abundan los fragmentos de encinares con pinos y algunas otras especies arbóreas intercaladas, además de una rica flora de epífitas, particularmente orquídeas (Wolf y Santiago, 1998; Hágsater et al., 2005).

Stelis zootrophionoides es una especie con distribución geográfica restringida, previamente solo conocida de la Reserva Ecológica Cerro Huitepec, en el municipio San Cristóbal de Las Casas. En esta área crece como epífita sobre acumulaciones de humus en los troncos de Quercus laurina Bonpl. (Fagaceae), en un bosque de niebla de encino y pino, a 2,554 m de altitud (Ramos-Castro et al., 2012).

En el presente estudio se informa del descubrimiento de nuevas poblaciones y formas florales (basadas en la coloración) de $S$. zootrophionoides, se modela su distribución potencial, y se determina la estructura de su población más vulnerable. Con ello se evalúa su riesgo de extinción.

\section{Materiales y métodos}

Toma de datos. De 2011 a 2013 se descubrieron nuevas poblaciones de Stelis zootrophionoides. Cada una de estas poblaciones fue georreferenciada con ayuda de un equipo GPS (Garmin, eTrex 10), y de cada una de ellas se describió la coloración de las flores de los individuos que las componían, como una estimación de la variación morfológica y genética que exhibe la especie.

Modelación de la distribución potencial. Se utilizó el Algoritmo Genético para la Predicción de un conjunto de Reglas (GARP, por sus siglas en inglés; Stockwell y Peters, 1999; Anderson et al., 2003) mediante su implementación para PC DesktopGARP (Scachetti-Pereira, 2002; http://www.nhm. ku.edu/desktopgarp/). Este algoritmo ha mostrado tener una excelente capacidad de predicción de la distribución poten- cial de las especies (Anderson et al., 2002; Peterson et al., 2002; Peterson et al., 2008), particularmente para especies endémicas, con pocas localidades conocidas, raras o difíciles de encontrar (Sánchez-Cordero et al., 2001). Funciona de manera iterativa, con la selección de un conjunto de reglas o condiciones ambientales, una evaluación y prueba de ésta, su eventual incorporación o rechazo al modelo y así sucesivamente. Estas reglas, se refieren a la relación que tienen las localidades de recolecta con las variables ambientales y resultan un modelo preliminar del nicho ecológico y una predicción inicial de la distribución potencial de la especie (Sánchez-Cordero et al., 2001; Benito de Pando y Peñas de Giles, 2007).

Como registros de presencia se utilizaron las cinco localidades hasta ahora conocidas para $S$. zootrophionoides (cuatro de ellas aquí reportadas como nuevas localidades para la especie), 19 variables ambientales actuales elaboradas a alta resolución para México (Cuervo-Robayo et al., 2014; http://idrisi.uaemex.mx/) y tres variables topográficas (U.S Geological Survey Hydro.1k; http://usgs.gov). Ambos conjuntos de coberturas (ambientales y topográficas; Tabla 1) se delimitaron para el estado de Chiapas. Se generaron 100 modelos con los siguientes parámetros de elaboración: 0.01 de límite de convergencia, 1,000 iteraciones, debido al bajo número de localidades se utilizó $100 \%$ para construir

Tabla 1. Variables ambientales y topográficas empleadas para generar el modelo de distribución potencial de Stelis zootrophionoides (Orchidaceae).

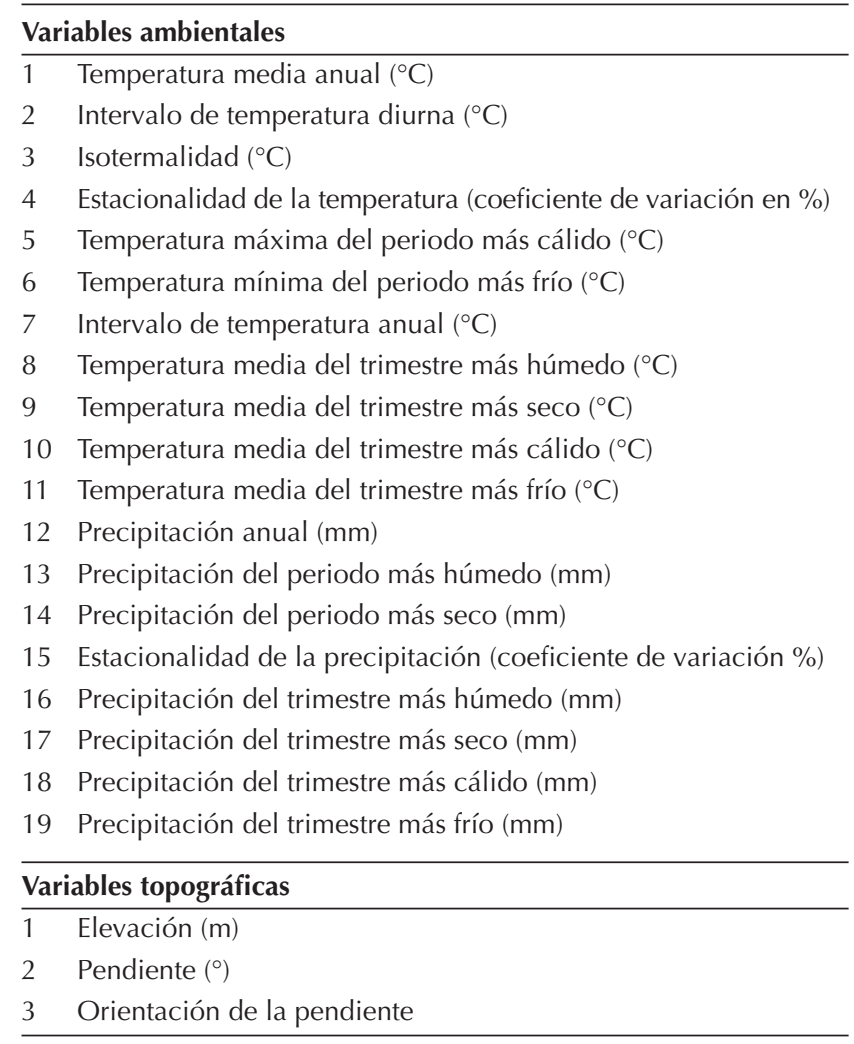



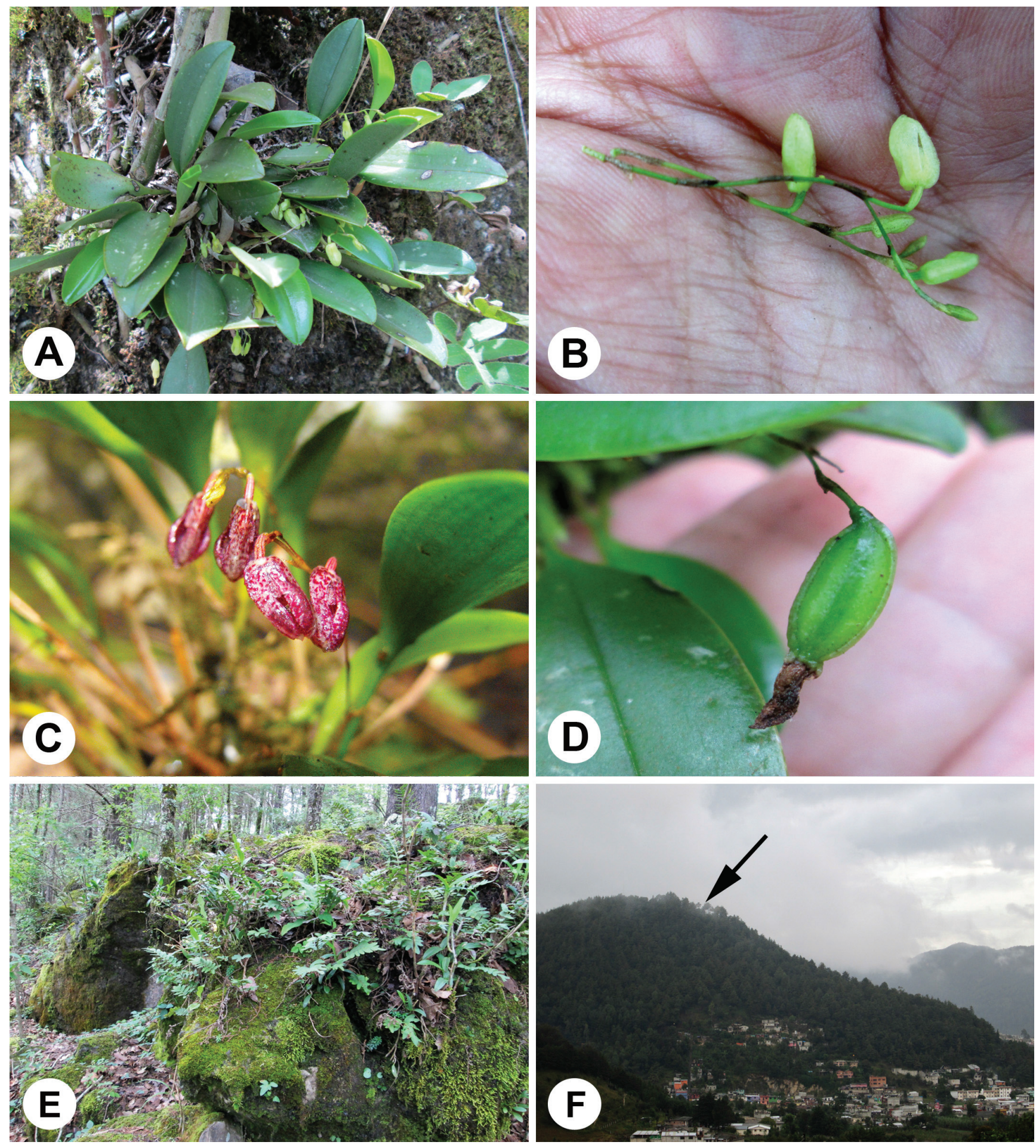

Figura 1. A. Planta de la forma "albina" de Stelis zootrophionoides (Orchidaceae), creciendo como rupícola en el Cerro Santa Cruz, San Cristóbal de Las Casas, Chiapas, México; B. Flores de la forma "albina”; C. Flores de la forma variegada en la Reserva Chichihuistán, Teopisca, Chiapas, México; D. Fruto; E. Una de las rocas sobre las que crece S. zootrophionoides en Cerro Santa Cruz; F. Expansión de la mancha urbana sobre el Cerro Santa Cruz (la flecha señala la posición aproximada de la población de S. zootrophionoides de la forma "albina"). (Autores: A, B, D, E, F. García-González A.; C. Martínez-Ovando E.). 
el modelo, una medida de omisión extrínseca, un umbral de omisión duro con $10 \%$ de error de omisión en los modelos generados, un umbral de error de comisión del $50 \%$. Se empleó la opción Best subset para obtener los 10 mejores modelos (cuyos valores del error de omisión fueran bajos y los valores de error de comisión moderados). A fin de obtener un mapa boleano se utilizó como línea de corte los píxeles donde existía un consenso de ocho modelos, establecidos a través de una prueba de $\chi^{2}\left(\chi^{2}=4.628 ; P<0.031\right)$. Finalmente el modelo se ajustó exclusivamente al área estimada.

Estatus de riesgo de Stelis zootrophionoides. Se empleó el Método de Evaluación del Riesgo de Extinción para plantas en México (MER-Plantas; SEMARNAT, 2010), el cual no asigna valores éticos o de conservación (Tambutti et al., 2001), y se puede aplicar a cualquier especie de la flora silvestre de México (Valverde et al., 2009), basándose en la evaluación de su índice de rareza (IR) y de su índice de impacto antropogénico (IIA). El IR incluye la valoración de tres criterios: A. Características de la distribución geográfica, B. Características del hábitat y C. Vulnerabilidad biológica intrínseca del taxón; por su parte el IIA evalúa un criterio: D. Impacto de la actividad humana sobre el taxón. Cada uno de estos criterios incluye varios subcriterios particulares. La evaluación de ambos índices permite determinar la categoría de riesgo en la que se encuentra la especie: en peligro de extinción (P), amenazada (A) o sujeta a protección especial (Pr) (SEMARNAT, 2010). La evaluación presentada aquí tomó en cuenta todas las poblaciones conocidas de $S$. zootrophionoides y las condiciones de su hábitat.

Caracterización de la población de Cerro Santa Cruz. Se decidió hacer una caracterización más detallada de la población de S. zootrophionoides localizada en Cerro Santa Cruz, teniendo en cuenta que esta es la que se encuentra en mayor riesgo. Esta población, es una de las más numerosas de las conocidas, sin embargo, todos los individuos se concentran en un área muy pequeña, existe una severa perdida de hábitat, está rodeada por la zona urbana de San Cristóbal de Las Casas, y representa la única localidad conocida para una de las formas florales aquí registradas. Se determinó la estructura de la población, dividiendo a los individuos en inmaduros o adultos (García-González y Riverón-Giró, 2014), se contó el número de frutos producidos por los individuos adultos y se determinó la posición cardinal en que se ubicaba cada individuo de la población. Como los individuos de esta población crecían sobre rocas, para conocer su posición cardinal se modificó la metodología propuesta por GarcíaGonzález y Riverón-Giró (2014). Se colocaron dos tramos de cuerda (4 m cada uno) cruzados y colocados sobre cada roca que ocupaba S. zootrophionoides, ubicando el punto de intersección lo más cercano posible al centro de las rocas. Con una brújula se determinó la posición cardinal en que se ubicaba cada individuo. Con los datos de orientación cardinal de las orquídeas, se realizó una prueba de Uniformidad Circular de Rayleigh, utilizando el programa Oriana 1.01. Como estadísticos descriptivos se aplicaron la media y el error estándar.

\section{Resultados}

Nuevas localidades y formas de Stelis zootrophionoides. Se reportan cuatro nuevas localidades para $S$. zootrophionoides, tres de ellas cercanas a la ciudad de San Cristóbal de Las Casas y otra en la Reserva Chichihuistán, en el municipio Teopisca, Chiapas (Figura 2A; Tabla 2). En dos de estas nuevas localidades se encontraron dos formas de la especie (basadas en la coloración de sus flores) no conocidas por la
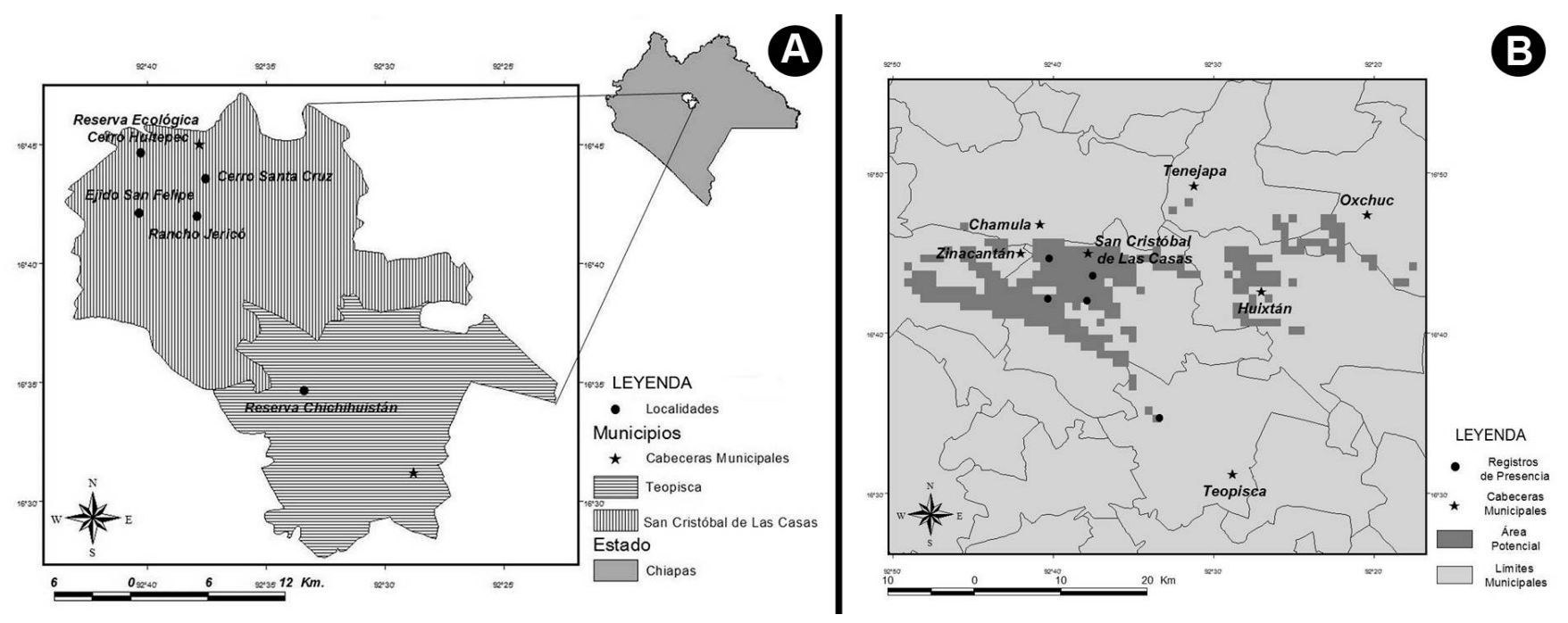

Figura 2. Localidades conocidas (A) y distribución potencial (B) de Stelis zootrophionoides (Orchidaceae) en Chiapas, México. 
Tabla 2. Datos de las poblaciones conocidas de la especie endémica mexicana Stelis zootrophionoides (Orchidaceae): color de las flores de cada población, hábitat donde se desarrollan, localidades y coordenadas geográficas de cada una, y altitud (m) a la que se encuentran. *Población de la localidad tipo de la especie (Ramos-Castro et al., 2012).

\begin{tabular}{|c|c|c|c|c|}
\hline Color de las flores & Hábitat & Localidad & Coordenadas & Altitud (m) \\
\hline *Púrpura & $\begin{array}{l}\text { *Bosque de niebla. Epífita en } \\
\text { acumulaciones de humus y } \\
\text { musgo, sobre Quercus laurina }\end{array}$ & $\begin{array}{l}\text { *Reserva Ecológica Cerro Huitepec, } \\
\text { noroeste de la ciudad de San Cristóbal } \\
\text { de Las Casas (municipio San Cristóbal } \\
\text { de Las Casas) }\end{array}$ & 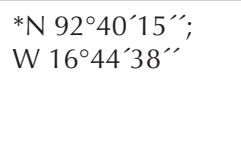 & $* 2,554$ \\
\hline Púrpura & $\begin{array}{l}\text { Bosque de encino. Epífita en } \\
\text { acumulaciones de humus y } \\
\text { musgo, sobre Quercus laurina }\end{array}$ & $\begin{array}{l}\text { Ejido San Felipe, suroeste de la ciudad } \\
\text { de San Cristóbal de Las Casas } \\
\text { (municipio San Cristóbal de Las Casas) }\end{array}$ & $\begin{array}{l}\text { N 92 } 42^{\circ} 18.77^{\prime \prime} ; \\
\text { W } 16^{\circ} 42^{\prime} 7.10^{\prime \prime}\end{array}$ & 2,405 \\
\hline $\begin{array}{l}\text { Variegada (púrpura } \\
\text { con manchas blancas) }\end{array}$ & $\begin{array}{l}\text { Acahual maduro de bosque } \\
\text { de niebla. Epífita en acumula- } \\
\text { ciones de humus y musgo, } \\
\text { sobre Cleyera theoides }\end{array}$ & $\begin{array}{l}\text { Reserva Chichihuistán, Ejido San Isidro } \\
\text { Chichihuistán (municipio Teopisca) }\end{array}$ & $\begin{array}{l}\text { N 92³3’22.47"'; } \\
\text { W 16³ }\end{array}$ & 1,953 \\
\hline No se conoce & $\begin{array}{l}\text { Bosque de pino-encino. } \\
\text { Rupícola en acumulaciones } \\
\text { de humus y musgo }\end{array}$ & $\begin{array}{l}\text { Rancho Jericó, sur de la ciudad de } \\
\text { San Cristóbal de Las Casas (municipio } \\
\text { San Cristóbal de Las Casas) }\end{array}$ & $\begin{array}{l}\text { N 92 } 37^{\prime} 53.62^{\prime \prime} ; \\
\text { W } 16^{\circ} 41^{\prime} 59.44^{\prime \prime}\end{array}$ & 2,337 \\
\hline $\begin{array}{l}\text { "albina" (amarillo- } \\
\text { verdoso) }\end{array}$ & $\begin{array}{l}\text { Bosque de encino y Cupressus } \\
\text { lusitanica. Rupícola en acumu- } \\
\text { laciones de humus y musgo }\end{array}$ & $\begin{array}{l}\text { Cerro Santa Cruz, este de la ciudad } \\
\text { de San Cristóbal de Las Casas } \\
\text { (municipio San Cristóbal de Las Casas) }\end{array}$ & $\begin{array}{l}\text { N 92 } 37^{\prime} 31.2^{\prime \prime} ; \\
\text { W } 16^{\circ} 43^{\prime} 33.54^{\prime \prime}\end{array}$ & 2,280 \\
\hline
\end{tabular}

ciencia para este taxón (Figura 1A, B, C; Tabla 2). Todos los individuos de la población de Cerro Santa Cruz exhiben flores de color amarillo-verdoso ("albinas"; Figura 1A, B; Tabla 2) y todos los de la Reserva Chichihuistán tienen flores variegadas, de color púrpura con manchas blancas (Figura 1C; Tabla 2). La coloración de las flores de la población de Rancho Jericó no se conoce, únicamente se han observado individuos con frutos.

Modelación de la distribución potencial. El modelo de distribución potencial para $S$. zootrophionoides, predice una extensión aproximada de $292 \mathrm{~km}^{2}$ (Figura 2B) en Los Altos de Chiapas e incluye las localidades donde la especie ha sido recolectada (Figura 2A, B).

Estatus de riesgo de Stelis zootrophionoides. Basado en la evaluación asignada a los índices planteados por el MERPlantas.

\section{I. Índice de rareza.}

Evaluación del criterio A: Características de la distribución geográfica.

Subcriterio A.1. Extensión de la distribución. De las cinco localidades conocidas de $S$. zootrophionoides, las más cercanas son Rancho Jericó y Cerro Santa Cruz (separadas por aproximadamente $3.01 \mathrm{~km}$ ), y la más aislada es la Reserva Chichihuistán (Figura 2A, B). El área del polígono que engloba las cinco localidades es de aproximadamente $60 \mathrm{~km}^{2}$, mientras que su distribución potencial es de menos de $300 \mathrm{~km}^{2}$ (Figura 2A, B). Por tanto, la amplitud de la distribución conocida y potencial de $S$. zootrophionoides resulta mayor a $1 \mathrm{~km}^{2}$ pero menor al $1 \%$ del Territorio Nacional (ca. 2,000,000 km²); 3 puntos.
Subcriterio A.2. Número de poblaciones o localidades conocidas existentes. Hasta el momento se han descubierto cinco localidades en el área de distribución de $S$. zootrophionoides, todas ellas muy próximas entre sí, por lo que no todas pueden ser discernibles en un mapa de una escala 1 : $4,000,000 ; 3$ puntos.

Subcriterio A.3. Número de provincias biogeográficas en las que se encuentra el taxón. De acuerdo al mapa de provincias biogeográficas de México (CONABIO, 1997), la especie solo se encuentra en una provincia biogeográfica, Los Altos de Chiapas; 3 puntos.

Subcriterio A.4. Representatividad de la distribución del taxón en el Territorio Mexicano. Stelis zootrophionoides es una especie endémica de México, por lo que su distribución en el país no es periférica o extralimital; 0 puntos.

\section{Valor total del Criterio A= 0.82 (9/11).}

Evaluación del criterio B: Características del hábitat.

Subcriterio B.1. ¿En cuántos tipos de vegetación se presenta? De acuerdo al mapa de vegetación potencial de México (Rzedowski, 1990), todas las localidades de S. zootrophionoides se encuentran en la región caracterizada como bosque de coníferas; $\mathbf{3}$ puntos.

Subcriterio B.2. ¿El taxón tiene un hábitat especializado? Stelis zootrophionoides crece preferentemente debajo del dosel, en lugares bien sombreados, con humedad atmosférica alta, en sitios con presencia de musgos y rodeadas de otras epífitas y/o rupícolas vasculares, lo cual favorece la acumulación de materia orgánica y el suministro de nutrientes para la germinación y crecimiento de los individuos. No 
obstante, su hábitat puede ser variable, ya que puede presentarse en un bosque de niebla, de encino, de pino-encino o de encino con Cupressus lusitanica Mill. (Cupressaceae; Tabla 2). La distribución restringida de la especie puede deberse a un conjunto de factores bióticos (p. ej: disponibilidad de polinizadores, hongos micorrícicos o árboles de sustrato) y/o abióticos (p. ej: fragmentación de los hábitats por agricultura, tala o urbanización). Sin embargo, no contamos con información precisa sobre la disponibilidad de sitios adecuados para $S$. zootrophionoides; 0 puntos.

Subcriterio B.3. ¿La permanencia de la población es dependiente de un hábitat primario? Si bien en tres de sus localidades $S$. zootrophionoides se encuentra en fragmentos de bosque relativamente conservados, que pueden considerarse como bosque primario, en las otras dos localidades crece en etapas intermedias de la sucesión del bosque (Tabla 2). En la Reserva Chichihuistán la especie crece en un acahual maduro derivado de bosque de niebla, mientras que en Cerro Santa Cruz crece en un bosque de Quercus crassifolia Bonpl. (Fagaceae) y C. lusitanica (Tabla 2), producto de una reforestación realizada posiblemente en la década de 1980 (Cervantes et al., 2008; Fernández-Pérez et al., 2013). Teniendo esto en cuenta, se puede afirmar que probablemente la especie prefiere bosques conservados, pero es tolerante al disturbio intermedio; 0 puntos.

Subcriterio B.4. ¿La permanencia de la población requiere de regímenes de perturbación particulares o está asociada a etapas transitorias en la sucesión? El establecimiento de $S$. zootrophionoides no parece requerir de un régimen de perturbación; 0 puntos.

Subcriterio B.5. Amplitud del intervalo altitudinal que ocupa el taxón. Las localidades conocidas de la especie se encuentran entre 1,953 y $2,554 \mathrm{~m}$ de altitud (Tabla 2), por lo que su intervalo altitudinal se extiende a $600 \mathrm{~m} ; 1$ punto.

Valor total del Criterio $B=0.44$ (4/9).

Evaluación del criterio $C$ : Vulnerabilidad biológica intrínseca.

Subcriterio C.1. Demografía.

Subcriterio C.1.1. Número total de individuos. De las poblaciones conocidas de $S$. zootrophionoides, las que cuentan con mayor número de individuos son la de Cerro Santa Cruz y la de Cerro Huitepec. No obstante, entre las cinco localidades, el número de individuos que hasta ahora han sido observados no supera los 100; 3 puntos.

Subcriterio C.1.2. Reclutamiento. No ha sido posible estimar la tasa de reclutamiento en todas las poblaciones conocidas del taxón, pero en la población de Cerro Santa Cruz se ha observado la presencia de individuos inmaduros; 2 puntos.
Subcriterio C.1.3. Atributos demográficos. A) ¿Hay evidencia de densodependencia en la reproducción? No se tienen evidencias de densodependencia en la reproducción; 0 puntos. B) ¿Hay clonalidad? Como en la mayoría de las orquídeas, S. zootrophionoides probablemente pueda propagarse clonalmente, pero no hay evidencia de ello; 1 punto. C) ¿Hay evidencia de decrecimiento de las poblaciones en el país? El taxón fue descrito recientemente (año 2012; Ramos-Castro et al., 2012) y se desconoce el estado de sus poblaciones antes de su descubrimiento; 0 puntos. D) ¿Hay evidencia de una varianza muy grande en la fecundidad? No existe información de la dinámica poblacional para el taxón y por ello no se dispone de datos sobre su fecundidad; es probable que los individuos reproductivos de mayor edad y biomasa acumulada tengan una mayor fecundidad, pero no hay datos que lo corroboren; 0 puntos. E) ¿El taxón es dioico, los individuos son dicógamos o autoincompatibles? La especie tiene flores funcionalmente hermafroditas, que solo dejan dos aberturas laterales muy pequeñas como entradas para polinizadores muy especializados, por lo que cabría esperar que fueran autocompatibles, pero no se dispone de información sobre su sistema reproductivo; 0 puntos. F) ¿La floración es sincrónica o gregaria? El periodo de floración de la especie se extiende de julio a octubre, aunque la población de Cerro Santa Cruz presenta un pico de floración en el mes de agosto; 0 puntos. G) ¿El taxón produce pocos propágulos? Posiblemente S. zootrophionoides produzcan un número de propágulos similar al de otros miembros de su linaje, ya sea de forma vegetativa (ramets y genets) o sexual (semillas); 0 puntos.

Subcriterio C.2. Genética. No se dispone de información molecular para el taxón, por lo que no se evalúan los Subcriterios C.2.1. Variación molecular, y C.2.2. Estructura genética molecular.

Subcriterio C.2.3. Cantidad de variación genética. Se ha observado que los individuos reproductivos dentro de una población son constantes en la coloración de sus flores, mientras que entre poblaciones se presentan diferencias en este rasgo (Tabla 2, Figura 1A, B, C). Por tanto, se estima de manera indirecta que la variación genética entre las poblaciones probablemente es alta, lo que podría indicar efecto fundador y estructuración genética en las poblaciones, las que posiblemente no se están entrecruzando. No se ha identificado el ataque de patógenos, por lo que no se puede utilizar este parámetro en la evaluación; 0 puntos.

Subcriterio C.2.4. Nivel de diferenciación entre poblaciones. La especie fue originalmente descrita a partir de una población de flores púrpuras concoloras (Ramos-Castro et $a l ., 2012)$ y se conocen poblaciones con otras dos formas florales ("albinas" y variegadas; Figura 1B, C; Tabla 2). Estas formas de coloración parecen ser constantes dentro de 
cada población, lo cual sugiere una estructura y diferenciación genética entre ellas, aunque hacen falta estudios para verificarlo; 1 punto.

Subcriterio C.3. Interacciones bióticas especializadas. Subcriterio C.3.1. ¿El taxón requiere una "nodriza" para su establecimiento? No existe información u observaciones que indiquen que $S$. zootrophionoides requiera una "nodriza" para su establecimiento; 0 puntos.

Subcriterio C.3.2. ¿El taxón requiere un hospedero o forófito específico? Las poblaciones de Ejido San Felipe y Cerro Huitepec crecen como epífitas sobre $Q$. laurina, mientras que la de la Reserva Chichihuistán crece sobre Cleyera theoides (Sw.) Choisy (Theaceae) (Tabla 2), por lo que no se observa una especificidad estricta del taxón por crecer sobre una especie de forófito en particular. No obstante, a nivel de población, los individuos solo crecen sobre una especie de árbol (Tabla 2). Por otra parte, los individuos de las poblaciones de Cerro Santa Cruz y Rancho Jericó crecen como rupícolas (Tabla 2); 0 puntos.

Subcriterio C.3.3. ¿El taxón requiere un polinizador específico? No se conoce el polinizador de esta orquídea. Sin embargo, en la familia Orchidaceae, uno de los grupos con más ejemplos de polinización por moscas es Pleurothallidinae (Neyland et al., 1995; Luer, 2000; Pridgeon et al., 2001). Generalmente las flores de este grupo poseen caracteres típicos de miofilia tales como, forma radial, labelo con manchas, pequeñas proyecciones, lóbulos fusionados, y hendeduras, que en algunos casos pueden ser muy reducidas (van der Pijl y Dodson, 1969; Christensen, 1994). Estudios en varios miembros de Pleurothallis, indican que los polinizadores son dípteros de las familias Chlorophidae y Phoridae, con una alta especificidad en las especies que visitan (Borba y Semir, 2001; Borba et al., 2001a, b). En un trabajo previo sobre autoecología de Stelis (S. argentata Lindl.) no se logró determinar su polinizador, aunque se observó actividad de moscas de la familia Drosophilidae (Christensen, 1992). En S. zootrophionoides, el tamaño, coloración y forma de las flores sugiere un sistema de polinización muy especializado dirigido a la atracción de pequeños dípteros de tamaño similar a Drosophila, los cuales deben ingresar por las diminutas aberturas dispuestas lateralmente entre los sépalos; 1 punto.

Subcriterio C.3.4. ¿El taxón tiene un dispersor específico? No, como en la mayoría de las orquídeas, las semillas son dispersadas principalmente por el aire (Arditti y Abdul Ghani, 2000; Hágsater et al., 2005) o también pueden ser transportadas en el plumaje de las aves (Arditti y Abdul Ghani, 2000); 0 puntos.

Subcriterio C.3.5. ¿El taxón presenta mirmecofilia obligada? No; 0 puntos.
Subcriterio C.3.6. ¿El taxón presenta dependencia estricta de la micorriza? Como la mayoría de las orquídeas, $S$. zootrophionoides necesariamente tiene que asociarse con hongos micorrícicos para poder germinar y desarrollarse (Jersáková y Malinová, 2007; Mujica-Benítez, 2007; Otero et al., 2007, Chung et al., 2011); 1 punto.

Subcriterio C.3.7. ¿El taxón sufre una afectación importante por depredadores, patógenos? En la población de Cerro Santa Cruz se encontró un fruto dañado por un herbívoro no identificado, sin embargo, de forma general en las poblaciones no se han observado afectaciones importantes provocadas por plagas, herbívoros o fitopatógenes, ni competencia con especies alóctonas o invasoras; $\mathbf{0}$ puntos.

Valor total del Criterio $C=0.39(9 / 23)$.

\section{II. Índice de impacto antropogénico.}

Evaluación del criterio D: Impacto de la actividad humana. Subcriterio D.1. ¿Cómo afecta al taxón la alteración antrópica del hábitat? Aunque en Los Altos de Chiapas aún existen manchones de las formaciones vegetales donde vive la especie (de tamaños variables y en diferentes estados de conservación y etapas sucesionales), en la mayoría de las localidades su hábitat consiste en fragmentos remanentes cercanos a asentamientos humanos, enfrentando una alta presión debido a la expansión de la mancha urbana, extracción de madera, apertura de campos agrícolas, extracción de leña y recolecta de plantas con fines comerciales y/o rituales (Matlack, 1997; Enríquez-Rocha y Rangel-Salazar, 2009; Calderón-Cisneros et al., 2012); 1 punto.

Subcriterio D.2. ¿Cuál es el nivel de impacto de las actividades humanas sobre el hábitat del taxón? Los factores antropogénicos arriba señalados presentan un fuerte impacto que afecta a todas las poblaciones del taxón; 3 puntos.

Subcriterio D.3. ¿Existe evidencia (mediciones, modelos o predicciones) que indique un deterioro en la calidad o extensión del hábitat como efecto de cambios globales o se prevé un cambio drástico en el uso del suelo? Actualmente en Los Altos de Chiapas es evidente el incremento en la tasa de conversión del bosque para agricultura, pastoreo de ovinos y establecimiento de nuevos asentamientos humanos (ParraVázquez et al., 1993; González-Espinosa et al., 1997). Este cambio en el uso del suelo ha provocado modificaciones en la estructura y composición florística (Ramírez-Marcial et al., 1998) y en la extensión de los remanentes forestales (Figueroa-Jáuregui et al., 2011). Figueroa-Jáuregui et al. (2011) analizaron los cambios de uso del suelo en la cuenca de San Cristóbal de Las Casas, en tres periodos, y encontraron que de 1975 a 1993 las tasas de cambio fueron positivas para bosque de pino (BP) (0.33) y zonas urbanas (ZU) (3.60), indicando aumento en sus coberturas, mientras que fueron negativas para las áreas de agricultura de temporal 
(AT) (-0.27), bosques de encinos (BE) (-0.43) y bosque de niebla $(\mathrm{BN})(-0.59)$, indicando pérdida en sus coberturas. De 1993 a 2000, las unidades en aumento fueron AT (0.98), $\mathrm{BP}(0.49)$ y nuevamente $\mathrm{ZU}(0.50)$; y hubo pérdida en BE $(-0.60)$ y $\mathrm{BN}(-3.46)$, donde se registró la mayor tasa de pérdida en los siete años. Por último, las tasas de cambio de 2000 a 2009 fueron negativas para AT (-1.39) y BP (-2.15), y positivas para $\mathrm{BE}$ (1.62), BN (3.26) y ZU (2.06). Las áreas agrícolas disminuyeron en el último periodo debido a que las comunidades indígenas más cercanas a la ciudad de San Cristóbal de Las Casas, abandonan parcialmente la agricultura para dedicarse a la confección y venta de artesanías, destinadas al floreciente mercado turístico en la ciudad. El abandono facilitó la regeneración de algunas áreas de bosque, pero aunado a esto, la zona urbana aumentó más del doble en 34 años (en los años estudiados paso de $4.13 \%$ de la superficie total de la cuenca a $9.7 \%$ ); 1 punto.

Subcriterio D.4. ¿Cuál es el impacto del uso sobre el taxón? El taxón no tiene importancia ornamental y no se dispone de información sobre usos tradicionales. No obstante, sus poblaciones podrían potencialmente verse afectadas por la extracción de plantas epífitas y/o rupícolas para el comercio local, pero no se dispone de evidencias de impacto por esta actividad; 0 puntos.

Subcriterio D.5. ¿El taxón es cultivado o propagado ex situ? A pesar de que el cultivo puede llegar a ser fuente de espe-

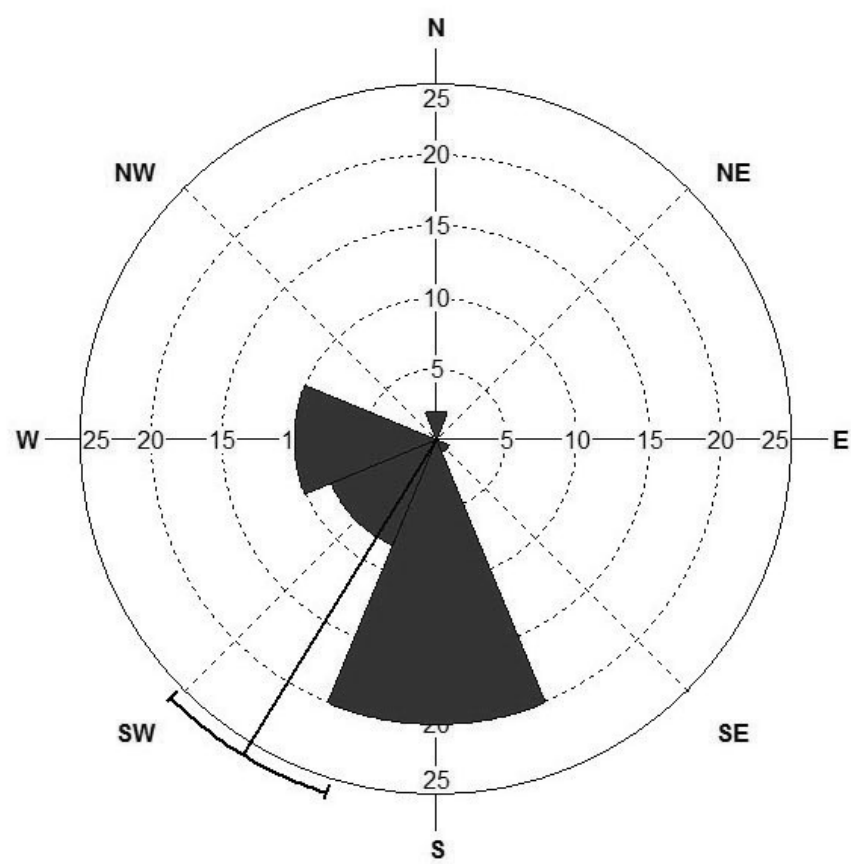

Figura 3. Diagrama de rosa de las orientaciones cardinales de Stelis zootrophionoides (Orchidaceae) en las rocas sobre las que crece en el Cerro Santa Cruz, San Cristóbal de Las Casas, Chiapas, México $(\mathrm{N}=41)$. Los pétalos más largos representan las orientaciones más frecuentes. Intervalos de confianza del $95 \%$. címenes en programas de conservación ex situ, actualmente el taxón no está siendo propagado; 0 puntos.

Valor total del Criterio $\mathrm{D}=0.5(5 / 10)$.

Sumatoria de valores obtenidos para los criterios A: 0.82 + B: 0.44 + C: 0.39 + D: $0.5=2.15$ puntos.

Caracterización de la población de Cerro Santa Cruz. Todos los individuos en Cerro Santa Cruz (forma "albina") crecen exclusivamente sobre dos rocas vecinas (Figura 1E), localizadas en un área de aproximadamente $40 \mathrm{~m}^{2}$. La población se ubica $20 \mathrm{~m}$ más abajo de la cima del cerro y a menos de $600 \mathrm{~m}$, hacia la base de la elevación, ya se encuentran viviendas pertenecientes a la ciudad de San Cristóbal de Las Casas (Figura 1F). La población está compuesta por 41 individuos, de los cuales 23 fueron inmaduros $(56.1 \%)$ y 18 adultos (43.9\%). De los individuos adultos, en ocho de ellos (44.8\%) se registró un total de 19 frutos (Figura 1D), con un promedio de $2.37 \pm 0.46$ frutos por planta. El mayor número de individuos de esta población crecen con orientación sur (20 individuos; Figura 3), existiendo diferencias significativas entre el número de individuos hacia las diferentes orientaciones cardinales (Uniformidad Circular de Rayleigh, $\mathrm{z}=20.986, P<0.001)$.

\section{Discusión}

Stelis zootrophionoides es una orquídea de distribución sumamente restringida que, paradójicamente, había pasado desapercibida en una zona extensamente explorada desde el punto de vista botánico (Quintana-Ascencio y González-Espinosa, 1993; Ramírez-Marcial et al., 1998; Wolf y Santiago, 1998). El doble hábito de crecimiento (epífito y rupícola) que presenta el taxón, es un fenómeno que llega a ser común en especies de otros géneros de orquídeas, que colonizan tanto árboles como rocas (p. ej: Encyclia, Epidendrum, Prosthechea, etc.), utilizando las mismas adaptaciones para vivir en ambos sustratos (Ackerman, 1995; Hágsater et al., 2005; Llamacho y Larramendi, 2005).

El modelo de distribución de especies es una herramienta que, junto con el desarrollo de los Sistemas de Información Geográfica (SIG), ha contribuido en los últimos años a la ampliación del conocimiento en diversos campos de investigación (biogeografía, conservación, ecología, paleoecología o climatología; Araujo y Guisan, 2006; Benito de Pando y Peñas de Giles, 2007). Para S. zootrophionoides el modelo resultó ser robusto $(P<0.031)$, se ajusta al rendimiento del algoritmo y no al azar, además, muestra un excelente desempeño, ya que no existen errores de omisión. Los datos obtenidos son confiables para identificar áreas idóneas para el establecimiento de la especie, en los municipios de Chamula, Zinacantán, Tenejapa, Huixtán y Oxchuc, aunque actualmente no existen registros de presencia en estos sitios. La información obtenida será muy útil a la hora de realizar verificaciones de campo y ampliar el estudio. 
Stelis zootrophionoides se puede considerar como una especie en peligro de extinción $(\mathrm{P})$, tanto por la puntuación obtenida con la suma de los cuatro criterios del MER-Plantas, como por vía directa, al tener menos de 500 individuos totales (SEMARNAT, 2010). De acuerdo a este resultado, es importante proponer la inclusión de la especie en la NOM059-SEMARNAT-2010, la norma que incluye a las especies silvestres mexicanas consideradas en alguna categoría de riesgo (SEMARNAT, 2010). Los valores asignados a cada criterio del MER-Plantas son susceptibles de modificación acorde con los resultados que aporten futuras investigaciones, no obstante, esto no implicaría necesariamente un cambio substancial en el estatus de conservación de $S$. zootrophionoides (Valverde et al., 2009). La especie presenta alta vulnerabilidad debido a las actividades antropogénicas que afectan sus hábitats, su distribución geográfica sumamente restringida, el pequeño número de localidades conocidas y su escaso número de individuos.

Por otra parte, la incorporación de nuevas localidades de este taxón difícilmente modificaría el valor del Subcriterio A.1, referente a la amplitud del área de distribución (Valverde et al., 2009). Por ejemplo, de encontrarse poblaciones de la especie en toda su área de distribución potencial $\left(292 \mathrm{~km}^{2}\right)$, esto sólo representaría aproximadamente $0.015 \%$ del Territorio Nacional.

En el caso de la población de S. zootrophionoides de Cerro Santa Cruz, se observa una marcada preferencia de los individuos por crecer con orientación sur $(48.8 \%$ de los individuos). Esto probablemente esté relacionado con la dirección y velocidad predominante de los vientos que impactan en el cerro por la ladera sur, donde se localizó la población (González-Hernández et al., 2007; Tremblay y Velázquez-Castro, 2009), y con que el microambiente (determinado por la luz y la humedad) hacia esa dirección sea más favorable para el establecimiento y crecimiento de los individuos (Mujica-Benítez, 2007).

La relación de individuos inmaduros/ individuos adultos en la población de Cerro Santa Cruz, y la presencia de plantas con frutos, puede indicar que el hábitat es adecuado para que esta orquídea desarrolle su ciclo de vida y que posiblemente la especie cuenta con suficientes polinizadores en el área (Ackerman y Montalvo, 1990). No obstante, esta última afirmación requiere estudios más específicos, ya que teniendo en cuenta la morfología floral de S. zootrophionoides (Ramos-Castro et al., 2012), parte de la producción de frutos también podría deberse a eventos de autopolinización. Las plantas inmaduras garantizan el reemplazo de las posibles bajas que ocurren en la población de forma natural (Mujica-Benítez, 2007), y el elevado porcentaje de individuos reproductores aseguran en gran medida el mantenimiento y la expansión de la especie hacia nuevos nichos disponibles, siendo la etapa adulta la más importante de la vida de las orquídeas (Zotz, 1998; Winkler y Hietz, 2001; García-Soriano, 2003; Mondragón, 2009). No obstante, son necesarios estudios que permitan conocer los parámetros demográficos de la población, éxito reproductivo, sistemas reproductivos y características genéticas, para evaluar su viabilidad.

La cercanía del Cerro Santa Cruz a la ciudad de San Cristóbal de Las Casas, su accesibilidad, la expansión de la zona urbana (principalmente por ocupación ilegal de terrenos), la deforestación de las zonas circundantes, la explotación de materiales pétreos en los cerros vecinos, la extracción de madera y el hecho de que el área no presente ninguna categoría de protección o manejo, ponen en grave peligro a esta población de $S$. zootrophionoides y a las demás especies con las que coexiste en su hábitat. Por otro lado, la extracción y comercio ilícito de orquídeas, bromelias y crasuláceas silvestres en la región, puede ser una amenaza potencial para la especie. Aunque no se ha observado la recolección y venta de individuos de S. zootrophionoides, la actividad es potencialmente peligrosa para está orquídea, fundamentalmente en época de floración, donde es más atractiva para su comercialización como miniatura, fenómeno observado con otras especies de orquídeas de porte y flores pequeñas, o bien, porque accidentalmente puede ser extraída junto con otras especies de orquídeas de mayor tamaño y/o flores más vistosas, que crecen alrededor de los individuos de $S$. zootrophionoides.

Considerando que $S$. zootrophionoides es una especie endémica de Los Altos de Chiapas y que de acuerdo a la evaluación del MER-Plantas se trata de una especie en peligro de extinción $(\mathrm{P})$, se hace necesario emprender y consolidar acciones para la conservación de las escasas poblaciones conocidas y de su hábitat. Si bien, al menos a nivel local, las poblaciones de Cerro Huitepec y de la Reserva Chichihuistán se ubican en áreas sujetas a protección, es fundamental proteger sus otras poblaciones, particularmente la de Cerro Santa Cruz, como única localidad donde se presenta la variante floral "albina".

\section{Agradecimientos}

A Claudia Ballesteros por la revisión y comentarios hechos a diversas partes del manuscrito. A Gerardo Salazar por sus oportunas recomendaciones. A los/las revisores anónimos, por sus acertadas observaciones. A la organización IDEA WILD por el equipamiento proporcionado, fundamental para la realización de este trabajo.

\section{Literatura citada}

Ackerman J.D. 1995. An orchid flora of Puerto Rico and the Virgin Islands. Memoir of the New York Botanical Garden, vol. 73, New York Botanical Garden, Nueva York.

Ackerman J.D. y Montalvo A.M. 1990. Short- and long-term limitations to fruit production in a tropical orchid. Ecology 71:263271.

Anderson R.P., Lew D. y Peterson A.T. 2003. Evaluating predictive models of species distributions: criteria for selecting optimal models. Ecological Modelling 162:211-232. 
Anderson R.P., Peterson A.T. y Gómez-Laverde M. 2002. Using niche-based GIS modeling to test geographic predictions of competitive exclusion and competitive release in South American pocket mice. Oikos 98:3-16.

Araújo M.B. y Guisan A. 2006. Five (or so) challenges for species distribution modelling. Journal of Biogeography 33:16771688 .

Arditti J. y AbdulGhani A.K. 2000. Tansley Review No. 110. Numerical and physical properties of orchid seeds and their biological implications. New Phytologist 145:367-421.

Benito de Pando B. y Peñas de Giles J. 2007. Aplicación de modelos de distribución de especies a la conservación de la biodiversidad en el sureste de la Península Ibérica. GeoFocus 7:100-119.

Borba E.L., Felix J.M., Solferini V.N. y Semir J. 2001a. Fly-pollinated Pleurothallis (Orchidaceae) species have high phenetic variability: evidence from isozyme markers. American Journal of Botany 88:419-428.

Borba E.L., Semir J. y Shepherd G.J. 2001b. Self-incompatibility, inbreeding depression and crossing potential in five Brazilian Pleurothallis (Orchidaceae) species. Annals of Botany 88:89-99.

Borba E.L. y Semir J. 2001. Pollinator specificity and convergence in fly pollinated Pleurothallis (Orchidaceae) species: a multiple population approach. Annals of Botany 88:75-88.

Calderón-Cisneros A., Soto-Pinto L. y Estrada-Lugo E. 2012. Entre la conservación del bosque y el crecimiento de la ciudad: las localidades rurales en el espacio periurbano del Huitepec en San Cristóbal de Las Casas, Chiapas, México. Estudios demográficos y urbanos 27:739-787.

Cervantes V., Carabias J. y Arriaga V. 2008. Evolución de las políticas públicas de restauración ambiental. En: CONABIO. Ed. Capital Natural de México, vol. III: Políticas Públicas y Perspectivas de Sustentabilidad, pp. 155-226, Comisión Nacional para el Conocimiento y Uso de la Biodiversidad, México D.F.

Christensen D.E. 1992. Notes on the reproductive biology of Stelis argentata Lindl. (Orchidaceae: Pleurothallidinae) in eastern Ecuador. Lindleyana 7:28-33.

Christensen D.E. 1994. Fly pollination in the Orchidaceae. En: Arditti J. Ed. Orchid biology: Reviews and perspectives VI, pp. 415-454. John Wiley \& Sons, Nueva York.

Chung M.Y., Nason J.D. y Chung M.G. 2011. Significant demographic and fine-scale genetic structure in expanding and senescing populations of the terrestrial orchid Cymbidium goeringii (Orchidaceae). American Journal of Botany 98:2027-2039.

CONABIO. 1997. Provincias biogeográficas de México, escala 1: 4,000,000. Comisión Nacional para el Conocimiento y Uso de la Biodiversidad. <http://www.conabio.gob.mx/informacion/ gis/layouts/rbiog4mgw.png $>$ (consultado 9 marzo 2014).

Cuervo-Robayo A.P., Téllez-Valdés O., Gómez-Albores M.A., Venegas-Barrera C.S., Manjarrez J. y Martínez-Meyer E. 2014. An update of high-resolution monthly climate surfaces for Mexico. International Journal of Climatology 34:2427-2437.

Dillon G. 1977. Respuesta de la American Orchid Society al proyecto de ley sobre especies en peligro. Sociedad Venezolana de Ciencias Naturales. Comité de Orquideología 16:14-18.

Enríquez-Rocha P.L. y Rangel-Salazar J.L. 2009. La Reserva Ecológica Huitepec. CONABIO. Biodiversitas 85:6-10.

Fay M.F. y Chase M.W. 2009. Orchid biology: from Linnaeus via Darwin to the 21st century. Annals of Botany 104:359-364.

Fernández-Pérez L., Ramírez-Marcial N. y González-Espinosa M. 2013. Reforestación con Cupressus lusitanica y su influencia en la diversidad del bosque de pino-encino en Los Altos de Chiapas, México. Botanical Sciences 91:207-216.

Figueroa-Jáuregui M.L., Ibáñez-Castillo L.A., Arteaga-Ramírez R., Arellano-Monterrosas J.L. y Vázquez-Peña M. 2011. Cambio de uso de suelo en la cuenca de San Cristóbal de Las Casas, México. Agrociencia 45:531-544.

García-González A. y Riverón-Giró F.B. 2014. Organización espacial y estructura de una población de Ionopsis utricularioides (Orchidaceae) en un área suburbana de Pinar del Río, Cuba. Lankesteriana 13:419-427.

García-Soriano R. 2003. Demografía, manejo y conservación de Artorima erubescens en Oaxaca, orquídea endémica del sur de México. Tesis de Maestría, Facultad de Medicina Veterinaria y Zootecnia, Universidad Autónoma de Yucatán, Mérida. 62 pp.

González-Hernández E., Raventós J., Mujica-Benítez E. y Bonet A. 2007. Estructura y ecología de la población del endemismo cubano Broughtonia cubensis (Orchidaceae), en Cabo San Antonio, Península de Guanahacabibes, provincia de Pinar del Río, Cuba. Lankesteriana 7:469-478.

González-Espinosa M., Ochoa-Gaona S., Ramírez-Marcial N. y Quintana-Ascencio P.F. 1997. Contexto vegetacional y florístico de la agricultura. En: Parra-Vázquez M.R. y Díaz-Hernández B.M. Eds. Los Altos de Chiapas: agricultura y crisis rural. Tomo I. Los recursos naturales, pp. 85-117, El Colegio de la Frontera Sur, San Cristóbal de Las Casas, Chiapas.

Hágsater E., Soto-Arenas M.A., Salazar-Chávez G.A., JiménezMachorro R., López-Rosas M.A. y Dressler R.L. 2005. Las orquídeas de México. Instituto Chinoín. México D.F.

IUCN y OSG. 2012. International Union for Conservation of Nature and Orchid Specialist Group. <http://www.iucn.org/ about/work/programmes/species/who_we_are/ssc_specialist_ groups_and_red_list_authorities_directory/plants/orchid_specialist_group/about_orchids/> (consultado 9 marzo 2014).

Jersáková J. y Malinová T. 2007. Spatial aspects of seed dispersal and seedling recruitment in orchids. New Phytologist 176:237240.

Llamacho J.A. y Larramendi J.A. 2005. Las orquídeas de Cuba. Greta, Sevilla.

Luer C.A. 2000. Systematics of Jostia, Andinia, Barbosella, Barbrodria, Pleurothallis, subgen. Antilla, subgen. Effusia, subgen. Restrepioidia, addenda to Lepanthes, Masdevallia, Platystele, Pleurothallis, Restrepiopsis, Scaphosepalum and Teagueia. Icones Pleurothallidinarum. Icones Pleurothallidinarum 20, Monographs in Systematic Botany from the Missouri Botanical Garden, vol. 79, Missouri Botanical Garden Press, San Luis.

Luer C.A. 2014. Stelis. En: Ackerman J.D. Coor. Orchid flora of the Greater Antilles, pp. 481-492, Memoirs of the New York Botanical Garden, vol. 109. The New York Botanical Garden Press, Nueva York.

Matlack G.R. 1997. Four centuries of forest clearance and regeneration in the hinterland of a large city. Journal of Biogeography 24:281-295.

Mondragón D. 2009. Population viability analysis for Guarianthe aurantiaca, an ornamental epiphytic orchid harvested in Southeast México. Plant Species Biology 24:35-41.

Mujica-Benítez E. 2007. Ecología de las orquídeas epífitas Broughtonia cubensis (Lindley) Cogniaux, Dendrophylax lindenii (Lindley) Bentham et Rolfe y Encyclia bocourtii Mujica et Pupulin en el Cabo San Antonio, Península de Guanahacabibes, Cuba. Análisis espacio-temporal e implicaciones del impacto 
de un fenómeno atmosférico severo. Tesis de Doctorado, Facultad de Ciencias, Universidad de Alicante, Alicante. 302 pp.

Neyland R., Urbatsch L.E. y Pridgeon A.M. 1995. A phylogenetic analysis of subtribe Pleurothallidinae (Orchidaceae). Botanical Journal of the Linnean Society 117:13-28.

Otero J.T., Flanagan N.S., Herre E.A., Ackerman J.D. y Bayman P. 2007. Widespread mycorrhizal specificity correlates to mycorrhizal function in the neotropical, epiphytic orchid Ionopsis utricularioides (Orchidaceae). American Journal of Botany 94:1944-1950.

Parra-Vázquez M.R., Nahed-Toral J., Soto-Pinto M.L., GarcíaAguilar M.C. y García-Barrios L.E. 1993. El sistema ovino Tzotzil de Chiapas: I. Dinámica del manejo integral. Agrociencia, Serie Recursos Naturales Renovables 3:79-97.

Peterson A.T., Ortega-Huerta M.A., Bartley J., Sánchez-Cordero V., Soberón J., Buddemeier R.H. y Stockwell D.R.B. 2002. Future projections for Mexican faunas under global climate change scenarios. Nature 416:626-628.

Peterson A.T., Papes M. y Soberón J. 2008. Rethinking receiver operating characteristic analysis applications in ecological niche modelling. Ecological Modelling 213:63-72.

Pridgeon A.M., Solano R.R. y Chase M.W. 2001. Phylogenetic relationships in Pleurothallidinae (Orchidaceae): combined evidence from nuclear and plastid DNA sequences. American Journal of Botany 88:2286-2308.

Quintana-Ascencio P.F. y González-Espinosa M. 1993. Afinidad fitogeografica y papel sucesional de la flora leñosa de los bosques de pino-encino de Los Altos de Chiapas, México. Acta Botanica Mexicana 21:43-57.

Ramírez-Marcial N., Ochoa-Gaona S., González-Espinosa M. y Quintana-Ascencio P.F. 1998. Análisis florístico y sucesional en la Estación Biológica Cerro Huitepec, Chiapas, México. Acta Botanica Mexicana 44:59-85.

Ramos-Castro S.E., Castañeda-Zárate M., Solano-Gómez R. y Salazar G.A. 2012. Stelis zootrophionoides (Orchidaceae: Pleurothallidinae), a new species from Mexico. PLoS One. 7:e48822.

Rzedowski J. 1990. Vegetación Potencial, escala 1: 4,000,000, IV.8.2. Atlas Nacional de México, Sección Naturaleza, vol. II. Instituto de Geografía, Universidad Nacional Autónoma de México, México. <http://www.conabio.gob.mx/informacion/ gis/layouts/vpr4mgw.png > (consultado 9 marzo 2014).

Sánchez-Cordero V., Peterson A.T. y Escalante-Pliego P. 2001. El modelado de la distribución de especies y la conservación de la diversidad biológica. En: Hernández H.M., García-Alderete A.N., Álvarez F. y Ulloa M. Comp. Enfoques contemporáneos para el estudio de la biodiversidad, pp. 359-380, Ediciones Científicas Universitarias, Fondo de Cultura Económica, Academia Mexicana de Ciencias A.C., Instituto de Biología, Universidad Nacional Autónoma de México, México D.F.

Scachetti-Pereira R. 2002. DesktopGarp: a software package for biodiversity and ecologic research. University of Kansas Biodiversity Research Center, E.U. <http://www.nhm.ku.edu/desktopgarp/> (consultado 3 marzo 2014).
SEMARNAT. 2010. Norma Oficial Mexicana NOM-059-SEMARNAT-2010. Protección ambiental-Especies nativas de México de flora y fauna silvestres-Categorías de riesgo y especificaciones para su inclusión, exclusión o cambio-Lista de especies en riesgo. Secretaría de Medio Ambiente y Recursos Naturales. Diario Oficial de la Federación, segunda sección, 30 de diciembre de 2010. <http://www.profepa.gob.mx/innovaportal/file/435/1/NOM_059_SEMARNAT_2010.pdf> (consultado 3 marzo 2014).

Solano-Gómez R. 2010. Dos especies nuevas de Pleurothallidinae (Orchidaceae) para México. Lankesteriana 9:447-454.

Solano-Gómez R. 2011. Three new species of Stelis (Orchidaceae) for México. Brittonia 63:325-333.

Solano-Gómez R. 2014. Three new species of Stelis (Orchidaceae; Pleurothallidinae) from México. Phytotaxa 158:255-264.

Solano-Gómez R. y Salazar G.A. 2007. A new species of Stelis (Orchidaceae, Pleurothallidinae) from Guerrero, México. Revista Mexicana de Biodiversidad 78:253-256.

Soto-Arenas M.A., Hágsater E., Jiménez-Machorro R., SalazarChávez G.A., Flores-González R. y Ruiz-Contreras I. 2007. Las Orquídeas de México: Catalogo Digital (CD-ROM). Instituto Chinoín, México D.F.

Stockwell D.R.B. y Peters D. 1999. The GARP modelling system: problems and solutions to automated spatial prediction. International Journal of Geographical Information Science 13:143-158.

Tambutti M., Aldama A., Sánchez O., Medellín R. y Soberón J. 2001. La determinación del riesgo de extinción de especies silvestres en México. Gaceta Ecológica 61:11-21.

Tremblay R.L. y Velázquez-Castro J. 2009. Circular distribution of an epiphytic herb on trees in subtropical rain forest. Tropical Ecology 50:211-217.

Valverde P.L., Zavala-Hurtado J.A., Jiménez-Sierra C., RendónAguilar B., Cornejo-Romero A., Rivas-Arancibia S., López-Ortega G. y Pérez-Hernández M.A. 2009. Evaluación del riesgo de extinción de Mammillaria pectinifera, cactácea endémica de la región de Tehuacán-Cuicatlán. Revista Mexicana de Biodiversidad 80:219- 230.

van der Pijl L. y Dodson C.H. 1969. Orchid flowers. Their pollination and evolution. University of Miami Press, Coral Gables.

Winkler M. y Hietz P. 2001. Population structure of three epiphytic orchids (Lycaste aromatica, Jacquiniella leucomelana, and $J$. teretifolia) in a Mexican humid montane forest. Selbyana 22:27-33.

Wolf J.H.D. y Santiago T. 1998. Diversidad de epífitas vasculares en un paisaje fragmentado en Los Altos de Chiapas, Chiapas, México. El Colegio de la Frontera Sur. Informe final SNIB-CONABIO proyecto No. B060. Comisión Nacional para el Conocimiento y Uso de la Biodiversidad, México D.F. <http://www. conabio.gob.mx/institucion/proyectos/resultados/InfB060.pdf> (consultado 28 abril 2014).

Zotz G. 1998. Demography of the epiphytic orchid, Dimerandra emarginata. Journal of Tropical Ecology 14:725-741.

Recibido: 15 de julio de 2014

Aceptado: 10 de octubre de 2014 